\title{
Whims of an Accreting Young Brown Dwarf: Exploring Emission Line Variability of 2MASSW J1207334-393254
}

\author{
Alexander Scholz, Ray Jayawardhana, \& Alexis Brandeker ${ }^{3}$ \\ Department of Astronomy \& Astrophysics, University of Toronto, 60 St. George Street, \\ Toronto, Ontario M5S3H8, Canada
}

\begin{abstract}
We report the first comprehensive study of emission line variability in an accreting young brown dwarf. We have collected 14 high-resolution optical spectra of 2MASSW J1207334-393254 (M8), a likely member of the nearby 8-millionyear-old TW Hydrae association with a recently identified planetary mass companion, in three observing runs between 2005 January-March on Magellan Clay telescope. These spectra show a variety of emission lines that are commonly seen in classical $\mathrm{T}$ Tauri stars. $\mathrm{H} \alpha$ line in particular shows dramatic changes in shape and intensity in our dataset, both on timescales of several weeks and several hours. In spectra from late-January, the line is relatively weak and only slightly asymmetric. Spectra from mid- and late-March show intense, broad (10\% width $\sim 280 \mathrm{~km} \mathrm{~s}^{-1}$ ) and asymmetric $\mathrm{H} \alpha$ emission, indicative of on-going disk accretion. Based on empirical diagnostics, we estimate that the accretion rate could have changed by a factor of $5-10$ over $\sim 6$ weeks in this brown dwarf, which may be in the final stages of accreting from its disk. March spectra also reveal significant 'quasi-periodic' changes in the $\mathrm{H} \alpha$ line profile over the course of a night, from clearly double-peaked to nearly symmetric. These nightly profile changes, roughly consistent with the brown dwarf's rotation period, could be the result of a redshifted absorption feature coming into and out of our line of sight; when the profile is double-peaked we may be looking into an accretion column, flowing from the inner disk edge on to the central object, indicating that the accretion is probably channelled along the magnetic field lines. Our findings provide strong support for the magnetospheric accretion scenario, and thus for the existence of large-scale magnetic fields, in the sub-stellar regime. We also present the first high-resolution optical spectrum of SSSPM J1102-3431 (M8.5), which has recently been identified as another likely sub-stellar member of the TW Hydrae association. Its emission lines are relatively narrow and fairly symmetric, suggesting that it is accreting only very weakly, if at all.
\end{abstract}


Subject headings: stars: formation, low-mass, brown dwarfs - line: profiles, formation — accretion, accretion disks — planetary systems — circumstellar matter - stars: individual (2MASSW J1207334-393254, SSSPM J1102-3431)

\section{Introduction}

Over the past few years, it has become increasingly clear that young brown dwarfs undergo a $\mathrm{T}$ Tauri phase, remarkably similar to that of their stellar counterparts. Dusty disks appear to be common around young brown dwarfs, as indicated by near- and midinfrared excess (e.g., Natta et al. 2002; Jayawardhana et al. 2003; Mohanty et al. 2004). Spectroscopic signatures of disk accretion, such as broad, asymmetric $\mathrm{H} \alpha$ emission lines, are now seen in objects down to nearly the Deuterium-burning limit (e.g., Jayawardhana, Mohanty, \& Basri 2002, 2003; Natta et al. 2004; Mohanty, Jayawardhana, \& Basri 2005). Irregular large-amplitude photometric variations, observed in some young brown dwarfs, may be related to accretion as well (Scholz \& Eislöffel 2004). A number of objects near and below the sub-stellar boundary also show forbidden emission lines, usually associated with jets and winds in T Tauri and Class I sources (e.g., Fernández \& Comerón 2001; Barrado y Navascués, Mohanty, \& Jayawardhana 2004; Natta et al. 2004; Barrado y Navascués \& Jayawardhana 2004).

Gizis (2002) identified the brown dwarf 2MASSW J1207334-393254 (hereafter 2M1207) as a likely member of the nearby $~ 8-$ Myr-old TW Hydrae association, and reported strong $\mathrm{H} \alpha$ emission in a low-resolution spectrum, possibly indicative of disk accretion. Jayawardhana et al. (2003) did not find significant L'-band $(3.8 \mu \mathrm{m})$ excess from it, but Sterzik et al. (2004) detected mid-infrared excess, a situation analogous to two other stellar members of TW Hydrae (Jayawardhana et al. 1999). Mohanty, Jayawardhana \& Barrado y Navascués (2003; hereafter MJB03) obtained high-resolution spectra of 2M1207, which showed broad asymmetric $\mathrm{H} \alpha$ as well as other strong Balmer and He I lines, bolstering the case for on-going accretion. These findings drew widespread attention to 2M1207 as a brown dwarf with a long-lived accretion disk that could serve as a useful benchmark for studies of disk evolution

\footnotetext{
${ }^{1}$ aleks@astro.utoronto.ca

2rayjay@astro.utoronto.ca

3 brandeker@astro.utoronto.ca
} 
in the sub-stellar regime. Recently, Chauvin et al. (2004, 2005) have found a likely planetary mass companion to 2M1207; the pair may have formed as a relatively wide brown dwarf binary.

Gizis \& Bharat (2004) noted significant differences in the equivalent width (EW) of $\mathrm{H} \alpha$ between their own 2001 measurements and those of Kirkpatrick et al. (unpublished) and MJB03. These hints of variations, together with the broad interest in 2M1207, inspired us to investigate possible accretion variability in this system. Here we report dramatic changes in $\mathrm{H} \alpha$ emission seen in a series of high-resolution spectra taken between 2005 JanuaryMarch. We also present the first high-resolution spectrum of SSSPM J1102-3431 (hereafter SSSPM1102), another likely sub-stellar member of the TW Hydrae association, recently identified by Scholz et al. (2005).

\section{Observations and spectral analysis}

We obtained spectra of 2M1207 in three observing runs with the echelle spectrograph MIKE at the Magellan Clay 6.5-m telescope on Las Campanas. In the first run in January 2005 and the third run in March 2005, we used no binning and 0".35 slit, which gives a resolution of $R \sim 60000$. Integration times are between 900 and $1800 \mathrm{sec}$. The second run, also in March 2005, was carried out with $2 \times 2$ binning and 1".0 slit, providing $R \sim 20000$. The exposure times in this run are $3 \times 600 \mathrm{sec}$. Our secondary target SSSPM1102 was observed on 2005 March 16 with $3 \times 1200 \mathrm{sec}$ exposure time. Summary observing log for 2M1207 is given in Table 1 . MIKE is a double echelle spectrograph, consisting of blue and red arms, providing coverage from 3400 to $5000 \AA$ and 4900 to $9300 \AA$, respectively, in our configuration. All spectra were reduced using standard routines within IRAF. Consecutive exposures were co-added to improve the signal-to-noise ratio.

The spectra of 2M1207 show a variety of emission lines. The most prominent features are the Hydrogen Balmer lines, from $\mathrm{H} \alpha$ down to $\mathrm{H} 10$ at $3798 \AA$. HeI emission lines are clearly seen at 5876,6678, and $7065 \AA$. In addition, we detect emission in $\mathrm{NaD}(5890+5895 \AA)$, OI

(7774 and $8446 \AA$ ), several H Paschen lines, and in $\mathrm{CaH}+\mathrm{K}(3968+3933 \AA)$. The distinctive emission line spectrum appears in all epochs, but the lines are considerably weaker in the January data compared to the March run. In the March spectra, the Balmer and HeI lines exhibit broad wings and are in most cases clearly asymmetric. In contrast, in the January data these lines are much narrower and more symmetric. We note that none of our spectra shows forbidden line emission in [OI] or [SII]. The LiI $6708 \AA$ absorption is detected in the co-added 2M1207 spectrum with an EW of $0.4 \pm 0.1 \AA$, in agreement with the value of 0.5 given by MJB03. Our second target SSSPM1102 exhibits similar emission features to 
2M1207, in particular H Balmer and HeI, but the lines are narrow and fairly symmetric (Fig. 1, last panel). In that sense, SSPM1102 shows a spectrum comparable to 2M1207 during the January run. Lithium is tentatively detected in SSPM1102, with an EW of $0.4 \pm 0.2 \AA$, consistent with membership in the TW Hydrae group.

In this Letter, we focus on the most prominent H Balmer features of 2M1207, particularly $\mathrm{H} \alpha$, which shows dramatic changes both in shape and intensity in our dataset. In Fig. 1 we present the complete time series of $\mathrm{H} \alpha$ profiles. In the three January spectra (first three panels), the line is relatively weak and only slightly asymmetric. The March spectra, however, show very intense and in most cases strongly asymmetric $\mathrm{H} \alpha$ features. Furthermore, the profile exhibits dramatic, quasi-periodic changes, from strongly double-peaked to nearly symmetric with only a weak red 'shoulder', over the course of a night. The profile shapes of $\mathrm{H} \alpha$ in the March spectra can be understood as a superposition of a broad emission peak and a shallower absorption feature. The absorption component is clearly redshifted, with an offset of $40-60 \mathrm{~km} \mathrm{~s}^{-1}$ (see Table 1). Absorption and emission are both variable; the absorption component disappears and re-appears on timescales of about 1 day.

For the $\mathrm{H} \alpha$ emission line, which is the dominant feature in the red part of the spectrum, we determined the full width at $10 \%$ of the peak (hereafter $10 \%$ width, see Table 1 ). In addition, we measured line fluxes for prominent features by integrating the spectrum after subtracting the continuum. In the absence of absolute flux calibrations, these line fluxes can only be compared to values measured in the same spectrum. In Table 1 we list the flux ratio between the $\mathrm{H} \beta$ and the $\mathrm{H} \alpha$ Balmer lines. The results are given in Table 1 . The same measurements were also carried out for SSSPM1102.

\section{Geometry of the system}

Many of the emission lines seen in the spectrum of 2M1207 are typical indicators for ongoing accretion, and are frequently observed in classical T Tauri stars (CTTS). Perhaps the most intriguing feature is the redshifted absorption component in the $\mathrm{H} \alpha$ profile, which shows clear changes within a few hours. Redshifted absorption is often seen in the emission lines of CTTS, such as AA Tau, BP Tau (Johns \& Basri 1995), DN Tau (Muzerolle, Hartmann, \& Calvet 1998), and TW Hya (Alencar \& Batalha 2002). These features are clear signatures of infalling material, and are usually explained in a scenario in which the line of sight points into a funnel flow, and we see the hot spot(s) against the inflowing gas and not against the photosphere. According to the magnetospheric accretion models of Hartmann, Hewett, \& Calvet (1994), red absorption is a strong function of the inclination $i$ between the rotational axis and the line of sight, and should only appear if the system has $i \gtrsim 60^{\circ}$, i.e. when the disk 
is seen close to edge-on. Indeed, it has been shown that $\mathrm{T}$ Tauri stars with red absorption have disks at fairly high inclinations, e.g. AA Tau $\left(i=75^{\circ}\right.$, Bouvier et al. 1999) and BP Tau $\left(i=60-70^{\circ}\right.$, Simon, Dutrey, \& Guilloteau 2000). Therefore, it is likely that 2M1207 is also seen with an inclination $i \gtrsim 60^{\circ}$, i.e. close to edge-on.

In this scenario, one would expect the red absorption to vary on timescales similar to the rotation period, as a consequence of hot spots co-rotating with the object. MJB03 determined a projected rotational velocity $\left(v \sin i\right.$ ) of $13 \mathrm{~km} \mathrm{~s}^{-1}$ for $2 \mathrm{M} 1207$, which corresponds to an upper period limit of $25 \mathrm{~h}$, assuming a radius of $\sim 0.27 R_{\odot}$ for $0.03 M_{\odot}$ and $10 \mathrm{Myr}$ (Chabrier et al. 2000). ${ }^{4}$ If the system is seen at high inclination, the rotation period is in the range of one day. Thus, the red absorption feature in 2M1207 is indeed variable on timescales of the rotation period. Since this is difficult to explain for a system seen close to face-on, this provides further evidence for our given interpretation. The reported lack of $K-L$ nearinfrared colour excess in 2M1207 (Jayawardhana et al. 2003) may also be consistent with a disk at high inclination, though alternate explanations, such as grain growth or an inner disk hole, are also plausible.

As pointed out in Sec. 2, 2M1207 shows the complete Balmer series down to $3800 \AA$. In spectra with a nearly symmetric $\mathrm{H} \alpha$ profile, the line fluxes in the higher Balmer lines tend to be increased relative to $\mathrm{H} \alpha$ (Table 1 ). This tendency provides additional evidence for our given interpretation of the profile changes: If $\mathrm{H} \alpha$ is double-peaked, the absorption dominates, and we see on average cooler gas than in the symmetric case, where the hot spot is unobscured.

We conclude that the most straightforward explanation for the shape and variations of the $\mathrm{H} \alpha$ profiles is a high inclination, implying that we see the disk close to edge-on. This explains both the presence of strong redshifted absorption as well as its variation on timescales of the rotation period. At times when we see strong redshifted absorption, we may be looking into an accretion column. The fact that we probably see rotational modulation of this absorption provides evidence for inhomogenuous rather than spherical accretion, as expected in a scenario where the accretion is channeled by the magnetic field. Thus, our findings strongly support magnetospheric accretion in brown dwarfs.

\footnotetext{
${ }^{4}$ The radius, and thus the upper period limit, keeps roughly constant for reasonable uncertainties in mass $(30 \%)$ and age (5-20 Myr).
} 


\section{Variable Accretion in 2M1207}

By comparing the spectra from January with those from March, it is obvious that the mass accretion process on 2M1207 is significantly variable. There is a distinct change in the line profiles (see Fig. 1) and an increase in the $10 \%$ width, which is shown as a function of time in Fig. 2. In the March spectra, the $\mathrm{H} \alpha$ profile is clearly dominated by accretion, with broad emission and additional redshifted absorption. The higher levels of the Balmer series and HeI are also broad and asymmetric, again indicating on-going accretion (Muzerolle et al. 2003). The $10 \%$ widths in March are substantially higher than $200 \mathrm{~km} \mathrm{~s}^{-1}$ (Fig. 2), which has been proposed as a useful empirical threshold between accreting and non-accreting systems (Jayawardhana, Mohanty, \& Basri 2003). In contrast, the three January spectra are significantly narrower, and only slightly asymmetric. In one January spectrum, weak blueshifted absorption is visible, usually an indication of outflowing material. Given the lack of forbidden line emission, this is the only evidence for wind in our spectra. The $10 \%$ width in January is only slightly larger than $200 \mathrm{~km} \mathrm{~s}^{-1}$, which implies that 2M1207 is only weakly accreting, if at all, at that time.

The $10 \%$ width of $\mathrm{H} \alpha$ can be used to estimate the accretion rate. Natta et al. (2004) calibrated the correlation between accretion rate and $10 \%$ width using objects for which the accretion rate was derived from continuum veiling. We now use their empirical relation to assess the accretion rate for 2M1207. For the three January spectra, we obtain an average value of $213 \pm 3 \mathrm{~km} \mathrm{~s}^{-1}$ for the $10 \%$ width. This corresponds to an accretion rate of $10^{-10.8 \pm 0.5} M_{\odot \mathrm{yr}^{-1}}$, where the error estimate takes into account both the scatter of the $10 \%$ widths and the uncertainty in transforming to accretion rates. In contrast, the spectra from March 2005 show a $10 \%$ width of $281 \pm 18 \mathrm{~km} \mathrm{~s}^{-1}$. This converts to an accretion rate of $10^{-10.1 \pm 0.7} M_{\odot} \mathrm{yr}^{-1}$. Although the accretion rates in January and March are comparable within the errors ${ }^{5}$, the $10 \%$ widths are higher in March by $32 \%$, which clearly points to a significant change in the accretion rate as well.

It is instructive to compare these results with previous spectroscopic observations of 2M1207. Gizis (2002) report very strong H $\alpha$ emission in two consecutive spectra for 2M1207 taken in 2001, with EWs $\sim 300 \AA$, corresponding to $10 \%$ widths in the range of $300 \mathrm{~km} \mathrm{~s}^{-1}$ (Muzerolle et al. 2003), i.e. comparable to our March data. On the other hand, MJB03 and Kirkpatrick et al. (unpublished, see Gizis \& Bharat 2004) have measured H $\alpha$ EWs of 28 and $42 \AA$, likely corresponding to $10 \%$ widths around $200 \mathrm{~km} \mathrm{~s}^{-1}$, i.e. similar to our January

\footnotetext{
${ }^{5}$ We note, however, that the uncertainty in the relation between accretion rate and $10 \%$ width is mainly caused by differences in individual objects, whereas we consider here only one particular object. Thus the difference between January and March accretion rates is more significant than the formal error suggests.
} 
spectra. Thus, the literature data support the strong variations in the $\mathrm{H} \alpha$ widths (and hence the accretion rate). From the available data we conclude that the accretion rate of 2M1207 changes by a factor of 5-10 on a timescale of six weeks. Strong variations in the accretion behaviour have been observed for T Tauri stars in the past. Basri \& Batalha (1990) find veiling variations in the range of an order of magnitude for several objects, e.g. RW Aur, AA Tau, DG Tau, which implies significant changes in the accretion rate (Muzerolle, Hartmann, \& Calvet 1998). The veiling of TW Hya is variable between 0.84 and 0.12 on timescales of a few months (Alencar \& Batalha 2002), again suggesting variability in the accretion rate. Here we confirm for the first time that brown dwarfs can behave in a very similar manner. Observations with better time sampling and longer baselines are required to obtain more precise constraints on the timescales and the extent of the accretion variability of $2 \mathrm{M} 1207$.

As already mentioned, our second target SSSPM1102 shows similar spectral features to 2M1207, in particular H Balmer lines. Its $\mathrm{H} \alpha 10 \%$ width is $194 \mathrm{~km} \mathrm{~s}^{-1}$, and the profile shows a weak red absorption component (see Fig. 1, last panel). Thus, according to the criteria of Jayawardhana, Mohanty, \& Basri (2003), SSSPM1102 is only weakly accreting, if at all, at the time of observation. The $\mathrm{H} \alpha$ profile shape and $10 \%$ width are similar to the January data of 2M1207, indicating an accretion rate of $\sim 10^{-11} M_{\odot} \mathrm{yr}^{-1}$ for SSSPM1102.

\section{Conclusions}

We have obtained a time series of 14 high-resolution spectra of the young, accreting brown dwarf 2M1207, which show a variety of emission lines confirming on-going accretion. These features are, however, significantly weaker in the January spectra in comparison to the March data. Particularly the $\mathrm{H} \alpha$ line is strongly variable in shape and width. The $10 \%$ width varies by $32 \%$ on a timescale of six weeks, indicating a change in the accretion rate by a factor of 5-10. In the high accretion state, the line profile changes from doublepeak to nearly symmetric on timescales of a few hours, with a redshifted absorption feature that disappears and re-appears on timescales of $\sim 1 \mathrm{~d}$, consistent with the brown dwarf rotation period. This profile variability can be explained in a scenario where the accretion disk is seen at a relatively low inclination with respect to the line of sight. In cases where we see a double-peaked $\mathrm{H} \alpha$ line, we may be looking into an accretion column, co-rotating with the object. Thus, the profile variability provides strong evidence for funneled rather than spherical accretion. Hence, these observations make a compelling case that large-scale magnetic fields play an important role in the accretion process of brown dwarfs.

We thank the Las Campanas Observatory staff for their assistance and the anonymous 
referee for helpful suggestions. This research was supported by an NSERC grant and University of Toronto startup funds to R.J.

\section{REFERENCES}

Alencar, S. H. P., Batalha, C. 2002, ApJ, 571, 378

Barrado y Navascués, D., Jayawardhana, R. 2004, ApJ, 615, 840

Barrado y Navascués, D., Mohanty, S., Jayawardhana, R. 2004, ApJ, 604, 284

Basri, G., Batalha, C. 1990, ApJ, 363, 654

Bouvier, J., et al. 1999, A\&A, 349, 619

Chabrier, G., et al. 2000, ApJ, 542, 464

Chauvin, G., et al. 2004, A\&A, 425, 29

Chauvin, G., et al. 2005, A\&A, in press (astro-ph/0504659)

Fernández, M., Comerón, F. 2001, A\&A, 380, 264

Gizis, J. E., Bharat, R. 2004, ApJ, 608, 113

Gizis, J.E. 2002, ApJ, 575, 484

Hartmann, L., Hewett, R., Calvet, N. 1994, ApJ, 426, 669

Jayawardhana, R., et al. 2003, AJ, 126, 1515

Jayawardhana, R., Mohanty, S., Basri, G. 2003, ApJ, 592, 282

Jayawardhana, R., Mohanty, S., Basri, G. 2002, ApJ, 578, 141

Jayawardhana, R., et al. 1999, ApJ, 521, 129

Johns, C., Basri, G. 1995, AJ, 109, 2800

Mohanty, S., Jayawardhana, R., Basri, G. 2005, ApJ, 626, 498

Mohanty, S., et al. 2004, ApJ, 609, 33

Mohanty, S., Jayawardhana, R., Barrado y Navascués, D. 2003 ApJ, 593, 109 [MJB03] 
Muzerolle, J., et al. 2003, ApJ, 592, 266

Muzerolle, J., Hartmann, L., Calvet, N. 1998, AJ, 116, 455

Natta, A., et al. 2004, A\&A, 424, 603

Natta, A., et al. 2002, A\&A, 393, 597

Scholz, A., Eislöffel, J. 2004, A\&A, 419, 249

Scholz, R.-D., et al. 2005, A\&A, 430, 49

Simon, M., Dutrey, A., Guilloteau, S. 2000, ApJ, 545, 1034

Sterzik, M. F., et al. 2004, A\&A, 427, 245 

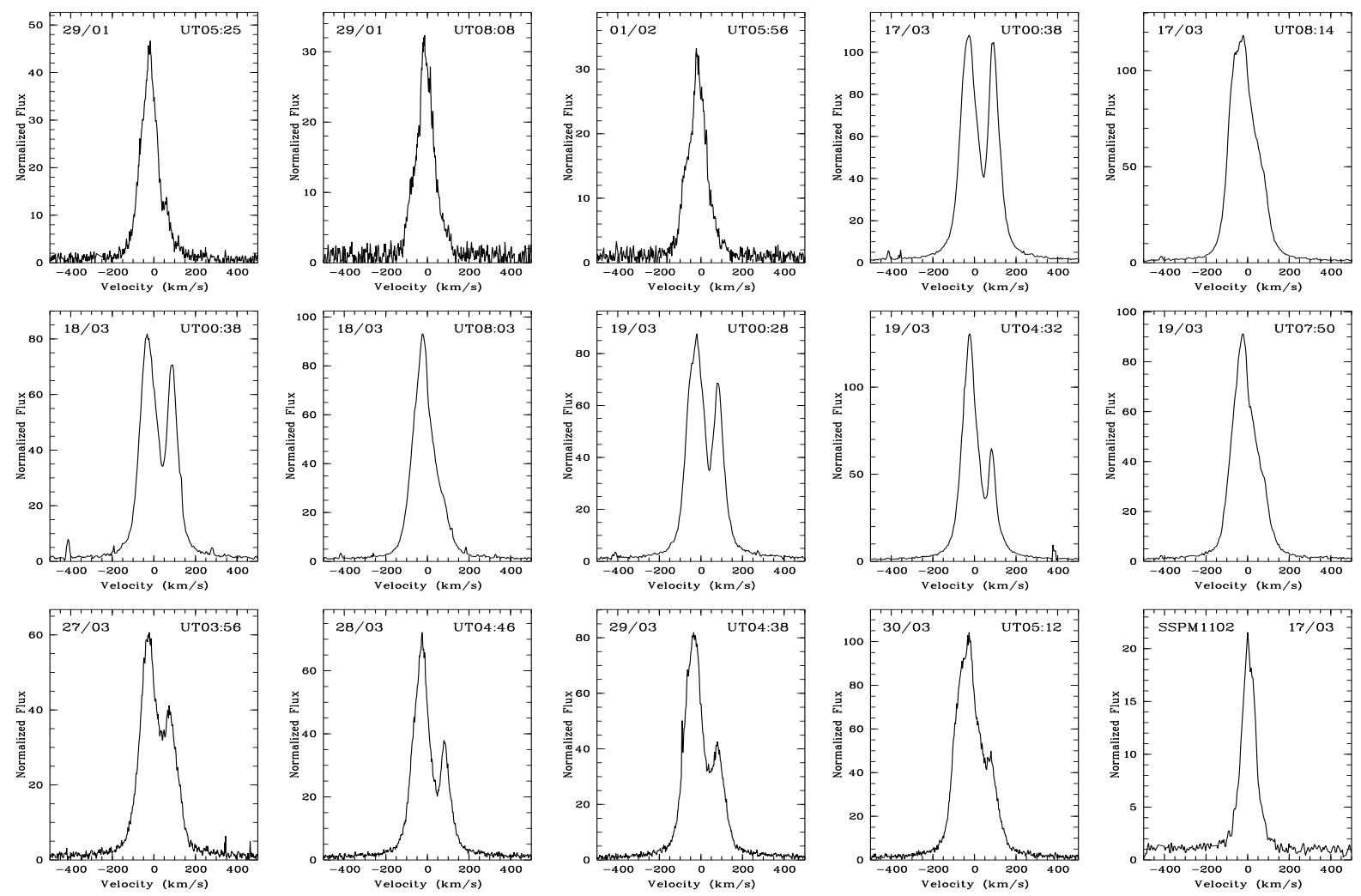

Fig. 1.- H $\alpha$ line profiles for 2M1207 (panels 1-14) and SSPM1102 (panel 15). The spectra of 2M1207 are ordered according to Table 1. All fluxes are normalized to the continuum. 


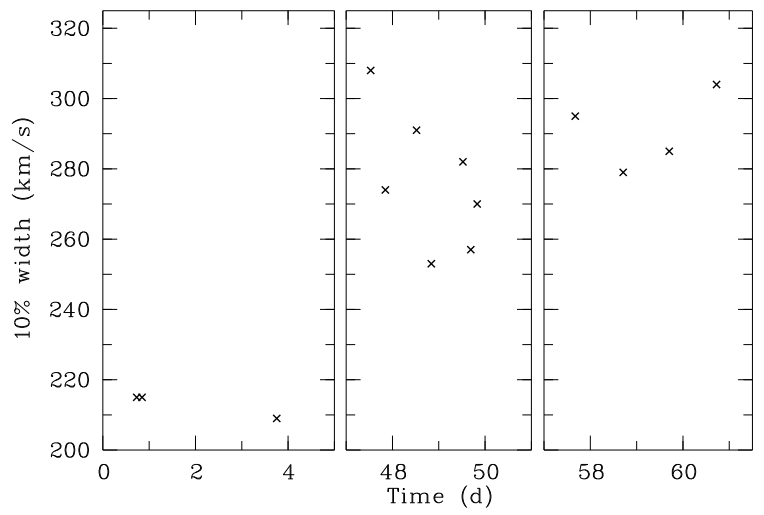

Fig. 2.- $10 \%$ width of $\mathrm{H} \alpha$ as a function of time (Julian Date minus 2453399.0). The three panels show the data from three different observing runs. A significant increase of the $\mathrm{H} \alpha$ width was observed between the first and the second run. 


\begin{tabular}{cccccc}
\hline \hline No. & $\begin{array}{c}\text { Date } \\
2005\end{array}$ & $\mathrm{UT}$ & $\begin{array}{c}\mathrm{H} \alpha 10 \% \\
\left(\mathrm{~km} \mathrm{~s}^{-1}\right)\end{array}$ & $\mathrm{H} \beta / \mathrm{H} \alpha$ & $\begin{array}{c}\Delta V_{\mathrm{Abs}} \\
\left(\mathrm{km} \mathrm{s}^{-1}\right)\end{array}$ \\
\hline 1 & $29 / 01$ & $05: 25$ & 215 & 0.30 & \\
2 & $29 / 01$ & $08: 08$ & 215 & 0.42 & \\
3 & $01 / 02$ & $05: 56$ & 209 & 0.38 & \\
4 & $17 / 03$ & $00: 38$ & 308 & 0.21 & 46 \\
5 & $17 / 03$ & $08: 14$ & 274 & 0.31 & \\
6 & $18 / 03$ & $00: 23$ & 291 & 0.14 & 40 \\
7 & $18 / 03$ & $08: 03$ & 253 & 0.30 & \\
8 & $19 / 03$ & $00: 28$ & 283 & 0.38 & 40 \\
9 & $19 / 03$ & $04: 32$ & 257 & 0.35 & 49 \\
10 & $19 / 03$ & $07: 50$ & 270 & 0.36 & \\
11 & $27 / 03$ & $03: 56$ & 295 & 0.23 & 41 \\
12 & $28 / 03$ & $04: 46$ & 279 & 0.22 & 47 \\
13 & $29 / 03$ & $04: 38$ & 285 & 0.25 & 41 \\
14 & $30 / 03$ & $05: 12$ & 304 & 0.27 & 59 \\
\hline \hline
\end{tabular}

Table 1: Observing log and line data for 2M1207. The last column contains the velocity offset of the red absorption component, if it is clearly detectable. The error for velocities is $\sim 10 \mathrm{~km} \mathrm{~s}^{-1}$, for flux ratios $\sim 10 \%$. 